\title{
El espacio en intervenciones para la prevención de dengue
}

\section{The space in interventions for dengue prevention}

\section{Carolina Ocampo}

Licenciada en Ciencias Biológicas. Becaria Doctoral del Consejo Nacional de Investigaciones Científicas y Técnicas (CONICET). Facultad de Ciencias Exactas y Naturales. Universidad de Buenos Aires. Intendente Güiraldes 2160 Ciudad Universitaria, (1428) Ciudad Autónoma de Buenos Aires, Buenos Aires, Argentina, caro.ocampo.mallon@gmail.com, ORCID https://orcid.org/0000-0002-1172-6910

Federico di Pasquo

Doctor en Ciencias Biológicas. Investigador Asistente del Consejo Nacional de Investigaciones Científicas y Técnicas (CONICET). Grupo de Filosofía de la Biología. Facultad de Ciencias Exactas y Naturales. Universidad de Buenos Aires. Intendente Güiraldes 2160 Ciudad Universitaria, (1428) Ciudad Autónoma de Buenos Aires, Buenos Aires, Argentina, dipasquof@yahoo.com.ar, ORCID https://orcid.org/0000-0001-5907-2437

Aníbal Carbajo

Doctor en Ciencias Biológicas. Investigador Independiente del Consejo Nacional de Investigaciones Científicas y Técnicas (CONICET). Grupo de Ecología de Enfermedades Transmitidas por Vectores. Instituto de Investigación e Ingeniería Ambiental. Universidad Nacional de San Martín. Campus Miguelete, 25 de Mayo y Francia, (1650) San Martín, Buenos Aires, Argentina, acarbajo@unsam.edu.ar, ORCID https://orcid.org/0000-0002-8792-7276

Recibido: 30 de diciembre 2019 || Aprobado: 15 de julio 2020

Resumen

El problema que abordamos se centra en la recuperación teórica del concepto de espacio en artículos científicos que tienen como pretensión el control o la prevención del dengue. En tanto la incidencia de esta enfermedad sigue creciendo desde su reaparición en Argentina, el control parece ser ineficiente. Ante la situación descripta cabe preguntarse cuáles podrían ser las razones del fracaso, y si entre ellas se presentan elementos epistémicos. Por lo tanto, el objetivo principal es indagar qué se considera por espacio en publicaciones académicas con interés en alguna intervención química de control de Aedes aegypti. Para ello realizamos una comparación analítica entre la noción de espacio como lugar y la noción de espacio según fue formulada por Milton Santos. Al respecto, hallamos que estas publicaciones consideran al espacio como lugar y que esta concepción elementista conlleva consecuencias negativas sobre el territorio.

Palabras clave: Conocimiento; Dengue; Epidemiologia; Geografía

\section{Abstract}

In our analysis we focus on the control of Aedes aegypti as a way to prevent dengue. The incidence of this disease is still growing since its reappearance in Argentina in 1998. Therefore, the control seem to be inefficient. In this context is relevant to evaluate the reasons of the failure and if there are epistemological elements contributing to it. Thus, the principal purpose is to inquire how the space is considered in academic publications with interest in chemical interventions of control of Aedes aegypti. For this purpose we make an analytical comparison between the space concept as place and the concept of space formulated by Milton Santos. In this regard, we find that these publications consider the space as place and that this elementist conception implies negative consequences over the territory.

Key words: Knowledge; Dengue; Epidemiology; Geography

Cita sugerida: Ocampo, C.; di Pasquo, F. y Carbajo, A. (2020). El espacio en intervenciones para la prevención del dengue. Estudios Socioterritoriales. Revista de Geografía, (28), 061. DOI: https://doi.org/10.37838/unicen/est.28-061 


\section{INTRODUCCIÓN}

Las intervenciones desarrolladas sobre los territorios infestados con Aedes aegypti, el mosquito vector de numerosos patógenos como el virus del dengue, están basadas en el conocimiento científico de las ciencias naturales y exactas. Conocimiento, usualmente dirigido a este insecto y a las distintas formas de eliminarlo. Asimismo, la construcción de este conocimiento y las preguntas de investigación descansan, entre otras cosas, sobre una forma específica de conocer el mundo y a su vez, sobre alguna concepción de la sociedad a la cual se intenta intervenir (García, 2000; Luz, 1997; di Pasquo, Klier, Busan y del Castillo, 2019). Por lo tanto, entre los numerosos saberes disponibles para construir soluciones es el conocimiento científico el que se privilegia. Y más aún, entre los saberes científicos disponibles, suele preponderarse el de las ciencias naturales y exactas. Es decir, la ciencia es la forma socialmente privilegiada e institucionalmente legitimada de producción de verdades (Luz, 1997). Una de las características de este saber experto, es que fragmenta las problemáticas y propone soluciones que visibilizan determinados riesgos (y omiten otros) ante los cuales es necesario prevenirse (Francese y Folguera, 2018). En general, en el problema del dengue, esta propuesta de solución aparece en forma de promesa a través de algún tipo de control de Aedes aegypti (Dantur Juri, Zaidenberg y Santana, 2013; Lucia, Harburguer, Licastro, Zerba y Masuh, 2009; Gürtler, Garelli y Coto, 2009). Así, la forma en que se construye la situación problemática implica los tipos de intervenciones que se llevan a cabo. En este marco queremos entender algunas de las características asumidas por el saber científico descripto.

Nuestro análisis se centra en explorar cómo se recupera teóricamente el concepto de espacio en artículos científicos que tienen como pretensión el control o la prevención del dengue. Esta enfermedad no tiene aún terapias antivirales específicas y la vacuna Dengvaxia $^{\text {NR }}$ aprobada contra las cepas virales en Argentina en el 2017 (ANMAT, 2017) no llegó a instrumentalizarse (WHO, 2017). En este sentido, la prevención queda tradicionalmente ligada al control vectorial y, en menor medida, a la vigilancia de casos probables. El control mencionado demuestra ser ineficiente ya que la enfermedad sigue creciendo en términos de incidencia desde su reaparición en el país en 1998. En 2016 se registró en Argentina una epidemia con 76.000 casos (MSAL, 2016a) siendo esta situación una tendencia para América, en tanto solo en el 2018 se incrementó el número de enfermos en 16 países (OPS, 2019).

Ante la situación descripta cabe preguntarse cuáles son las razones del fracaso en la prevención del dengue, y si entre ellas se presentan elementos epistémicos. Así, en este trabajo, analizamos la caracterización del espacio en ciertos estudios vinculados a la problemática del dengue, en tanto lo consideramos un elemento epistémico central. Asimismo, comprendemos que las fallas en el control del dengue no están sujetas exclusivamente a cómo se aborda el concepto teórico de espacio en las investigaciones sobre la enfermedad, ya que cualquier epidemia emerge como consecuencia de un entramado complejo de procesos. Por lo tanto, nuestro estudio solo pretende arrojar elementos para la reflexión en torno a los fracasos en la prevención en dengue.

El concepto de espacio es esencial en el estudio de las enfermedades transmitidas por vectores, en tanto ellas se abordaron, tradicionalmente, en asociación con condiciones ambientales (Silva, 1997). Por otra parte, el espacio interesa ya que las intervenciones de control implican actuar directamente en entornos humanos para eliminar al mosquito o 
bien, de forma indirecta, promoviendo una responsabilidad sobre el medio inherente al insecto (por ejemplo, sobre sus criaderos). El espacio, además, es un concepto básico en epidemiología, es decir, el lugar es uno de los descriptores centrales de las enfermedades (junto con tiempo y persona) (Gordis, 2015).

De esta forma, el espacio en la epidemiología dominante es absorbido por la categoría de lugar, entendida como la distribución geográfica de las personas en un tiempo determinado (Gordis, 2015). Esta noción se basa en una aprehensión estática, controlable y elementista del espacio que lo escinde de los seres que lo habitan. Cabe aclarar que no todas las corrientes en epidemiología se caracterizan por esta orientación acerca del espacio. De esta forma, encontramos, como ejemplo, a la epidemiología crítica (Breilh, 2010; 2015) o la epidemiología del paisaje (Meade, Earickson y Gesler, 1988) que rescatan otros acercamientos al espacio. En este sentido, como un posible contrapunto a la noción de lugar como la definimos, Milton Santos (2000) propone la categoría de espacio socialmente construido para enfatizar su existencia como una totalidad que se reconstruye una y otra vez, a través de los acontecimientos sociales que interaccionan con los objetos. Por lo tanto, considerando que se presenta una diversidad sobre las diferentes concepciones de espacio en la bibliografía especializada tanto de geografía como de epidemiología, nuestro objetivo principal, es analizar y problematizar qué se considera por espacio en ciertas publicaciones científicas con interés en alguna intervención química de control de Aedes aegypti. En este sentido, nuestras hipótesis son (a) que estas publicaciones consideran al espacio como lugar en sentido negativo; y (b) que esta concepción elementista de espacio conlleva consecuencias negativas sobre el territorio.

Para lograr el objetivo propuesto, se realizó un trabajo cualitativo. La elección descansó en que nuestro objetivo se dirige más a problematizar, específicamente, la concepción de espacio como lugar, que a reconocer por ejemplo, preponderancias o subordinaciones estadísticas sobre su uso. En este sentido, se seleccionaron artículos académicos que utilizaran de forma conjunta o aislada productos químicos (larvicidas y/o adulticidas) en experiencias a campo o de semi-campo. Es decir, de todos los posibles controles vinculados a Aedes aegypti (manejo ambiental, control biológico, químico, protección personal y educación para la salud) se seleccionó el químico en tanto este tipo de intervención es la más tradicional y antigua de todas las detalladas. Se usa desde la campaña de erradicación de Aedes aegypti a mediados del siglo XX en la cual se privilegió a los adulticidas, en especial el diclorodifeniltricloroetano (DDT), como método de eliminación (Raghavendra, Barik, Reddy, Sharma y Dash, 2011). Por otra parte, la intervención química resulta esencial para los controles focales de casos y es, por lo tanto, la medida de urgencia principal para detener las epidemias (MSAL, 2016b). Como consecuencia de su centralidad, en otros países se han cuestionado fuertemente los gastos públicos que genera sostener este tipo de aplicaciones y cuántos beneficios realmente reportan (Santos, 2003; Salinas-López, Soto-Rojas y Ocampo, 2018; Santos y Augusto, 2011).

A partir de buscadores académicos como SCOPUS y BVS se eligieron todos los trabajos de Argentina, realizados por investigadores argentinos. Para ello se introdujeron en los campos de búsqueda de título/resumen los términos libres en la siguiente combinación: "Argentina" AND "control" OR "prevención" AND "Aedes aegypti" OR "dengue". Estas primeras búsquedas se complementaron con la selección en PUBMED, SCOPUS, BVS y SCIELO de los artículos encontrados al introducir como términos libres "Argentina" AND "dengue". Se realizó una revisión sistemática de los trabajos recuperados por las búsque- 
das y se los clasificó de acuerdo a los diferentes abordajes de estudio sobre el dengue y/o la población de Aedes aegypti (ejemplo, modelado espacial de la enfermedad, control mixto sobre los mosquitos). La clasificación se logró mediante la lectura de los resúmenes de cada artículo. De esa selección se analizaron, de forma exploratoria, diez trabajos con alguna recomendación interventora sobre el territorio. Definimos las recomendaciones como todas aquellas propuestas explícitas de aplicación sobre un territorio de algún tratamiento que incluyera un producto químico y que fuese evaluado en el artículo científico. De los trabajos analizados, cinco incorporaban de forma única una aplicación química, y otros cinco presentaban intervenciones mixtas (por ejemplo: uso de un producto químico y manejo ambiental). La lista de los estudios seleccionados se detalla en la Tabla 1.

Tabla 1. Lista de artículos seleccionados para el análisis sobre la categoría de espacio

Año de publicación y autores

Artículos y revistas de publicación

Field evaluation of a smoke-generating formu2003 - Masuh, H.; De Licastro, S.A.; Lopez, lation containing beta-cypermethrin against the P.A.; Vega, C. y Zerba, E. dengue vector in Argentina. Journal of the American Mosquito Control Association, 19(1), 53-57.

Effectiveness of pyriproxyfen and diflubenzuron 2008 - Seccacini, E.; Lucia, A.; Harburguer, formulations as larvicides against Aedes aegypti. L.; Zerba, E.; Licastro, S. y Masuh, H. Journal of American Mosquito Control Association, 24(3), 398-403.

Patterns of Aedes aegypti (Diptera: Culicidae) in-

2009 - Garelli, F.M.; Espinosa, M.O.; Weinberg, D.; Coto, H.D.; Gaspe, M.S. y Gurtler, R.E.

festation and container productivity measured using pupal and Stegomyia indices in northern Argentina. Journal of Medical Entomology, 46(5), 11761186.

Efficacy of a new combined larvicidal-adulticidal 2009 - Lucia, A.; Harburguer, L.; Licastro, S.; Zerba, E. y Masuh, $\mathrm{H}$.

ultralow volume formulation against Aedes aegypti (Diptera: Culicidae), vector of dengue. Parasitology Research, 104(5), 1101-1107.

Effects of a five-year citywide intervention pro2009 - Gürtler, R.E.; Garelli, F.M. y Coto, H.D. gram to control Aedes aegypti and prevent dengue outbreaks in northern Argentina. PLoS Neglected Tropical Disease, 3(4), e427.

Water use practices limit the effectiveness of a temephos-based Aedes aegypti larval control program in northern Argentina. PLoS Neglected Tropical Diseases, 5(3), e991. berg, D.; Trinelli, M.A. y Gürtler, R.E.

A new strategy for Aedes aegypti (Diptera: Culicidae) control with community participation using a new fumigant formulation. Journal of Medical Entomology, 48(3), 577-583.

2011 - Harburguer, L.; Beltrán, G.; Gold berg, L.; Goldberg, L.; Zerba, E.; Licastro, S. y Masuh, $\mathrm{H}$.

The efficacy of a combined larvicide-adulticide in ultralow volume and fumigant canister formulations in controlling the dengue vector Aedes aegypti (Diptera: Culicidae) in Northwest of Argentina. Parasitology Research, 112(3), 1237-1246. 
Aedes aegypti (Diptera: Culicidae): evaluation of

2014 - Seccacini, E.; Juan, L.; Zerba, E. y Licastro, S. natural long-lasting materials containing pyriproxyfen to improve control strategies. Parasitology Research, 113(9), 3355-3360.
2019 - Rubio, A.; Cardo, M.V.; Carbajo, A.E. y Vezzani, D.
Assessment of combined tools and strategies for Aedes aegypti control with low environmental impact. Parasitology Research, 118(2), 411-420.

Fuente: elaboración personal

Asimismo, en los artículos seleccionados se analizó el modo de caracterizar al espacio, ya fuera de forma explícita o implícita, a través de las metodologías utilizadas. Dado que nuestro interés está en problematizar la concepción de espacio como lugar, recurrimos a una metodología comparada para visibilizar tanto aspectos positivos como negativos en dicha concepción. Al respecto, se realizó una comparación analítica (Sartori, 1994; 2011) entre la noción de espacio como lugar y la noción de espacio formulada por Milton Santos (espacio miltoniano), que fue recuperada en bibliografía especializada.

A continuación, introduciremos las nociones de espacio como lugar y espacio miltoniano que se discutirán en el resto del trabajo. En esta sección, nos detendremos en las particularidades de las nociones en discusión (mediante la comparación analítica sugerida) con la finalidad de facilitar la comprensión de los argumentos esgrimidos en el desarrollo siguiente. Así, en la tercera sección analizaremos, efectivamente, las caracterizaciones halladas sobre la noción de espacio en las publicaciones científicas seleccionadas. A su vez, ofreceremos una discusión acerca de la noción de espacio como lugar, utilizando el contrapunto generado con la noción de espacio miltoniano. Finalmente se comunicarán las conclusiones y algunas perspectivas.

\section{INTRODUCCIÓN A LAS NOCIONES UTILIZADAS}

En esta sección presentamos y comparamos las nociones de espacio escogidas: la noción de espacio como lugar y la noción de espacio miltoniano.

\section{NOCIÓN DE ESPACIO COMO LUGAR}

Los estudios epidemiológicos clásicos abordan como elementos centrales de análisis a las categorías de lugar, tiempo y persona (Gordis, 2015). En el contexto de esta epidemiología dominante, el espacio es comprendido como el área geográfica donde ocurren las enfermedades. Es decir, actúa como un recipiente natural que facilita el contacto entre las personas y los agentes etiológicos (Czeresnia y Ribeiro, 2000): "Una enfermedad puede transmitirse entre personas (transmisión directa) a través del contacto directo. La transmisión indirecta puede producirse a través de un vehículo común, como el aire o los suministros de agua contaminados (...)" (Gordis, 2015, p. 19). Como se puede notar en la cita, el espacio queda indirectamente referido como vehículo común, es decir como aquello que simplemente permite la llegada del patógeno a las personas. Por lo tanto, el espacio es considerado un lugar estático, aislado y a-histórico (Costa y Teixeira, 1999). Milton Santos (2000), por ejemplo, lo indica como paisaje, es decir un sistema material que es inmutable y que se encuentra compuesto de objetos. En este sentido, se trataría de la materialización de un instan- 
te de la sociedad (Zusman, 2002). De aquí que el lugar es aquello donde ocurre un acontecimiento, una simple localización o bien, una dimensión física que permanece escindida de los cuerpos (Monken et al., 2008). Otros autores lo describen como "un complejo de estímulos irradiados y exteriores al cuerpo" (Czeresnia y Ribeiro, 2000, p. 597), esto es, como un ente abstracto que puede ser fragmentado en diversas posibles causas de enfermedad. De esta forma, la descripción de lugar, en el mejor de los casos, queda detenida en enumerar factores que pueden asociarse con el evento de salud, es decir, en una visión elementista. Es importante destacar, a los fines de nuestro trabajo, que esta visión contribuye a entender los eventos de enfermedad a través de la desconexión de las relaciones que efectivamente tienen lugar entre los seres humanos y sus circunstancias. Por otro lado, se vuelve necesario aclarar que las concepciones de lugar desde la geografía también son numerosas, teniendo en algunos casos connotaciones positivas diferentes a las que se destacan en este estudio, como, por ejemplo, la de ámbito de la vida cotidiana, en el cual la identidad y lo simbólico son importantes (Ramírez Velázquez y López Levi, 2015).

\section{NOCIÓN DE ESPACIO MILTONIANO}

El concepto de espacio es particularmente problematizado por la llamada geografía crítica y la geografía radical que tienen como algunos de sus representantes más relevantes a Milton Santos, Henri Lefebvre y David Harvey. La geografía radical nace en 1960 en Estados Unidos y se presenta como un abordaje posible sobre las dinámicas de producción y dominación de la ciudad capitalista. A su vez, la geografía crítica aparece como una superación de la geografía radical, integrando sus logros con la reflexión acerca de otras formas de dominación urbana (Gintrac, 2013). Estas corrientes consideran que el espacio es una totalidad producida por la acción socialmente organizada de los seres humanos sobre un paisaje (Silva, 1997; Czeresnia y Ribeiro, 2000). Asimismo, las corrientes mencionadas se han sustentado fundamentalmente en la inclusión de elementos físicos dentro de las dinámicas de las relaciones sociales (Costa y Teixeira, 1999). Por lo tanto, el espacio no es solo un soporte, también genera significados y contribuye a la reproducción de mecanismos sociales, económicos y políticos (Zusman, 2002).

Milton Santos considera que "(...) el espacio está formado por un conjunto indisoluble, solidario y también contradictorio de sistemas de objetos y sistemas de acciones, no considerados aisladamente, sino como el contexto único en el que se realiza la historia" (Santos, 2000, p. 53). El espacio es, entonces, producto de la relación entre la materialidad de los objetos y todo lo que los crea, significa y transforma. Como esta relación se modifica a lo largo de la historia, la naturaleza del espacio es esencialmente social. Los objetos, o formas o fijos, son todos aquellos creados por el ser humano (hospitales, puertos, industrias) o, también, los considerados naturales (ríos, montañas, playas). Siguiendo con estos lineamientos, las acciones, o flujos o funciones, refieren a los movimientos, a las circulaciones de las personas y de las ideas (Santos, 2000; Monken et al., 2008).

Los elementos fijos, fijados en cada lugar, permiten acciones que modifican el propio lugar, flujos nuevos o renovados que recrean las condiciones ambientales y las condiciones sociales, y redefinen cada lugar. Los flujos son un resultado directo o indirecto de las acciones y atraviesan o se instalan en los fijos, modificando su significación y su valor, al mismo tiempo que ellos también se modifican. (Santos, 2000, p. 52) 
O en otro ejemplo:

A nuestro modo de ver, la cuestión a plantear es la de la propia naturaleza del espacio, formado, por un lado, por el resultado material acumulado de las acciones humanas a través del tiempo $y$, por otro lado, por las acciones actuales que le animan y que hoy le atribuyen un dinamismo y una funcionalidad. Paisaje y sociedad son variables complementarias cuya síntesis, siempre por rehacerse, viene dada por el espacio humano. (Santos, 2000, p. 88)

En relación con las dos últimas citas presentadas, se destaca que el espacio humano está siempre rehaciéndose a través de la definición mutua y continua de objetos y acciones. Así, las dinámicas sociales del espacio pueden conocerse buscando las localizaciones e identidades de los objetos y los usos e importancia que tienen estos para las acciones (Monken et al., 2008). Las acciones provienen de cubrir necesidades de diverso tipo, y son estas las que provocan la aparición de nuevos significados sobre los objetos. Por lo tanto, los objetos son productos de la acción social (Spinelli, 2016) y de igual forma estos nuevos objetos condicionan al propio sistema de acciones (Santos, 2000; Czeresnia y Ribeiro, 2000). En este sentido, Samaja (2003) contribuye a comprender la dinámica del espacio cuando menciona al medio ambiente como medio condicionante y condicionado de los sujetos individuales y colectivos.

Por lo tanto, el concepto de espacio miltoniano le puede agregar a la noción de lugar en epidemiología un gran valor simbólico y la necesidad de considerar los procesos constantes en él para aprehender su existencia:

Significa que el acontecimiento, para realizarse, se engarza en la forma (objeto) disponible más adecuada para que se realicen las funciones de que es portador. Por otro lado, desde el momento en que el acontecimiento se realiza, la forma, el objeto que lo acoge adquiere otra significación, proveniente de ese encuentro. En términos de significación y de realidad, uno no puede ser entendido sin el otro y, de hecho, uno no existe sin el otro. No pueden verse por separado. (Santos, 2000, p. 85)

El espacio es producto de una serie de decisiones basadas en la formación social, económica y política y estas, son consecuencia de ciertas relaciones de poder (Monken et al., 2008). En este sentido, la noción de territorio, en consonancia con el espacio miltoniano, es una construcción que expresa los modos de vida de los grupos sociales y que se carga de sentido al ser representado y apropiado/dominado (política, económica y culturalmente) por diferentes actores sociales (Spinelli, 2016; Faria y Bortolozzi, 2009). La palabra territorio es muy polisémica y puede ser definida distintamente según la visión política y teórica. Una de sus acepciones considera que el territorio es una parte del espacio (Monken et al., 2008) e involucra aquello que facilita o fuerza las interacciones entre sujetos, a partir de las cuales estos construyen sus identidades. Así, Milton Santos (2000) reconoce al territorio como sub-espacio en tanto indica que los recursos totales del mundo en cada momento histórico son distribuidos de diferente manera y están localmente combinados. Se entiende por recurso a toda posibilidad, material o no, de acción ofrecida a los humanos (Santos, 2000). Esto acarrea una diferenciación en el espacio total y confiere a cada lugar su especificidad y definición.

\section{EL ESPACIO EN LAS PUBLICACIONES CIENTÍFICAS ANALIZADAS}

\section{RECUPERACIÓN DE LA NOCIÓN DE LUGAR}

En esta sección analizamos las concepciones que aparecen en torno al espacio en las publicaciones académicas seleccionadas que utilizan algún control químico sobre Aedes aegypti. 
Inicialmente podemos destacar que no se hacen referencias explícitas hacia la noción de espacio. Usualmente es mencionado en términos de ambiente, en particular como un adjetivo descriptivo de algún producto químico (ambientalmente amigable). Por lo tanto, las referencias sobre qué es lo espacial se encontraron, fundamentalmente, en las propuestas metodológicas. Al respecto, presentamos cinco citas que evidencian la persistencia de la noción de espacio como lugar en las publicaciones académicas:

La fumigación se realizó mientras los residentes estaban ausentes de sus hogares. Quinientos cincuenta y dos casas se trataron entre el 30 de abril y el 4 de mayo del 2000. Se aplicó un pote fumígeno en la parte central de cada vivienda, dejando abiertas las puertas de los cuartos y de los armarios (Masuh et al., 2003, p. 54, traducción nuestra).

Dos áreas de aproximadamente 36 ha cada una, completamente separadas por áreas verdes, se usaron para tratamientos de ULV con permetrina $15 \%$ + piriproxifeno $3 \%$ (formulación $\mathrm{AL}$ ) y permetrina 15\% (formulación A). Una tercera área sin tratar se mantuvo como zona de control. Las tres áreas escogidas tenían características socioeconómicas similares y barreras naturales como ríos y largos espacios verdes actuando como zonas de buffer. (Lucia et al., 2009, p. 1102, traducción nuestra)

Las tasas de infestación se midieron antes, durante y después del tratamiento en todas las áreas. Se seleccionaron las áreas en base a características socioeconómicas similares (e.g. materiales de la casa, agua corriente, animales viviendo en o cerca de las viviendas) y la presencia de barreras naturales (como el río Salí) o áreas verdes (i.e vegetación natural y cultivos) que se consideraron como zonas buffer. (Dantur Juri et al, 2013, p. 1239, traducción nuestra)

Dentro de cada cementerio, se seleccionaron 35 hileras de tumbas consecutivas. El promedio de contenedores por hilera fue de $127 \pm 4$ ( $\min 52$, max 217 ) y el promedio en porcentaje de contenedores con agua fue de $55 \pm 8 \%$ ( $\min 40 \%$, max 76\%). Los seis tratamientos y el control se asignaron azarosamente entre las primeras siete hileras de tumbas, y este arreglo se repitió en el área remanente dando un total de cinco hileras por tratamiento en cada cementerio. El diseño minimizó los efectos de la heterogeneidad ambiental en el área de trabajo. (Rubio et al., 2019, p. 413, traducción nuestra)

La ciudad de Puerto Libertad se dividió en cuatro áreas con 200 casas cada una; las casas tenían el mismo tamaño y estaban distribuidas y espaciadas de igual forma dentro de cada área. Uno de cuatro posibles tratamientos se asignó al azar a cada área (...) En cada casa se aplicaron dos tabletas, una en el comedor/cocina y otra cerca de los dormitorios/baño, manteniendo abiertas todas las puertas de los cuartos y los armarios (...) De cada una de las tres áreas seleccionadas, se usó una zona central de $4 \times 4$ cuadras para llevar a cabo el monitoreo del vector. Cada cuadra tenía más o menos cinco casas separadas por un área verde, lo que hace un total de aproximadamente 100 casas. (Harburguer et al., 2011, p. 578, traducción nuestra)

En todas las citas analizadas se presentan ciertas similitudes que refieren a la noción de espacio como lugar, las cuales explicitaremos utilizando como ejemplo la última de ellas. En dicha cita, a la ciudad de Puerto Libertad, se la considera como un área pasible de ser dividida en casas, con el fin de obtener zonas limitadas en las cuales aplicar diferentes tratamientos. Las casas se proponen como ámbitos donde se pueden encontrar los mosquitos y por eso, susceptibles de control. Por otro lado, las zonas verdes se presentan como áreas de separación donde no se encontraría Aedes aegypti. Incluso dentro de las casas también se genera una separación y una homogeneización del espacio en zonas más pequeñas. De esta forma, el espacio es fragmentado y tratado simplemente como si fuera un área física.

Otros espacios, además de las casas, que se consideran relevantes para realizar tratamientos de aplicación de químicos, son los cementerios por ser considerados fuentes importantes de producción de mosquitos (véase la cuarta cita). Nuevamente, en estos casos, se indica la división del espacio para poder aplicar los tratamientos de forma 
azarosa. De forma adicional, se busca una reducción de la heterogeneidad del ambiente a partir de la aplicación rotativa de los tratamientos, es decir se persigue la homogeneidad para lograr cierta comparabilidad entre los tratamientos utilizados.

Otro aspecto relevante acerca de la noción de espacio como lugar, que puede derivarse de los trabajos analizados, es que los larvicidas y adulticidas implicados en los tratamientos son considerados estrictamente en términos de practicidad, eficiencia sobre el vector y seguridad en el uso. De esta forma se acepta que todos los recipientes que potencialmente pueden contener estadios larvales son criaderos. Es decir se produce, implícita o explícitamente, una homogeneización de los recipientes debido a la posible presencia del mosquito y a la vez, se eliminan sistemáticamente las posibles funcionalidades de ellos. En otras palabras, los recipientes son automáticamente transformados en criaderos de mosquitos, olvidando la multiplicidad de funciones que podrían poseer. Incluso en las excepciones a esto, no se destacan las particularidades de las funciones como parte de un sistema de objetos. Por ejemplo:

\section{Las potenciales ventajas operativas de estos materiales de larga duración conteniendo piriproxifeno sobre las formulaciones larvicidas disponibles comercialmente incluyen la larga duración de la efi- cacia, la posibilidad de convertir cualquier criadero en una trampa larvicida y una formulación fácil de manejar, y el hecho de que el piriproxifeno pueda ser usado en agua potable puede proveer una excelente alternativa a las ovitrampas letales. (Seccacini et al., 2014, p. 3359, traducción nuestra)}

En resumen, en los trabajos analizados el espacio es considerado simplemente como un conjunto de áreas divisibles y como un contenedor potencial de mosquitos adultos y/o estadios larvales, sin ninguna consideración analítica de la localización y el movimiento humano. En general, se incorpora el espacio como un conjunto de casas particulares con un cierto número de criaderos de Aedes aegypti, siendo los hogares y los recipientes fuertemente homogeneizados en torno a la presencia del mosquito. Cabe destacar que los cementerios (aun cuando descansan en la misma noción de espacio) son las únicas áreas públicas halladas en las publicaciones analizadas. En este punto del trabajo podemos sugerir una primera conclusión parcial: el acercamiento al espacio exclusivamente en términos de eficacia de un producto químico sobre la población del mosquito, en un área fragmentada, parece suponer un concepto simplificado de espacio como lugar. De aquí que no parece haber un reconocimiento de la dimensión simbólica del espacio, siendo este tratado únicamente como una dimensión física.

\section{LA COMPARACIÓN CON EL ESPACIO MILTONIANO}

En esta sección se discute el espacio como lugar, rastreado en las publicaciones académicas, a través de la comparación con la noción de espacio miltoniano (o socialmente organizado) de la geografía crítica.

De las publicaciones seleccionadas, podemos destacar que el problema del dengue parece quedar reducido a la efectividad de la intervención sobre la población del mosquito. A la vez, parecen desconocerse las acciones humanas, directa o indirectamente, involucradas en la formación y permanencia de los criaderos de Aedes aegypti. En algunos pocos casos se describen factores sociales, económicos o culturales que podrían estar influyendo en la aparición del mosquito pero solo en excepciones se opera sobre alguno de ellos como si fuera pasible de ser intervenido. Esas excepciones son la inclusión, como variables de impacto, del uso del agua por la comunidad y de las intervenciones previas realizadas por el investigador. Por lo tanto, en casi todos 
los casos analizados, el espacio parece quedar señalado como un simple conjunto de criaderos (sean estos naturales o artificiales).

En relación con lo anterior, aquellos recipientes susceptibles de ser criaderos son descriptos, únicamente, en términos ambientales (si están expuestos o no al sol, si tienen agua con pocos o muchos elementos orgánicos, entre otros) o bien, según la productividad de los estadios inmaduros del mosquito (se evalúa lo que ocurre antes y después de la aplicación del tratamiento solo en términos de la población de Aedes aegypti, excepto en Rubio et al. (2019) que analiza también una especie testigo). En este punto, la comparación con la noción de espacio miltoniano permite visibilizar una serie de inconvenientes. Por ejemplo, el que no se problematicen las razones de aparición y mantenimiento de los criaderos, lo cual supone una fuerte desconexión entre las acciones y los objetos. O en otras palabras, aparece el recipiente-criadero como si no se hubiese generado para cierto tipo de acciones y no hubiese sido, finalmente, definido por ellas. Por otra parte, se considera que el agregado del larvicida al criadero lo puede convertir en una trampa para el mosquito (Seccacini et al., 2014) pero, nuevamente, no se analizan otras variables vinculadas al uso de los recipientes que podrían evitar eso (como por ejemplo: la eliminación del material conteniendo larvicida en bebederos de animales o el tipo de recambio de agua en otros recipientes). De esta forma, aparece una visión en la cual los criaderos funcionan y pueden ser conocidos de forma aislada, ya no como elementos con funciones dentro de un conjunto. Es decir, los criaderos se piensan sin conexión entre ellos, sin vinculación con otros objetos del entorno y sin relación con los seres humanos, solo como meras colecciones.

Contrariamente a esta posición, aparecen ejemplos en los cuales se enfatiza la relación directa entre la falta de agua potable y las acciones humanas de acopio. En este sentido, la necesidad material determina la aparición de criaderos del mosquito que son relativamente estables y difíciles de controlar. Es decir, se vuelven notorios los heterogéneos hábitos del uso del agua que obligadamente adquieren las personas (Mastrangelo, 2013). Así, se incorpora una idea de interacción entre el entorno y el humano en donde los condicionamientos son mutuos. La práctica cultural, que parece generalizable, de mantener agua en recipientes, cobra otra significación en términos locales:

Las prácticas de uso de agua dependen de patrones culturales y disponibilidad de agua y los tres constituyen un conjunto complejo de factores afectando las dinámicas de transmisión de dengue. Las modificaciones ambientales, como la instalación de servicios confiables de agua de red, son una de las principales acciones para el control vectorial de dengue. (Garelli et al., 2011 , p. 8 , traducción nuestra)

Con relación a la última cita y volviendo sobre algunos lineamientos de la propuesta miltoniana, destacamos que la organización social del espacio explica, en buena medida, muchas de las relaciones de asociación encontradas entre el dengue y los criaderos del mosquito. En esta concepción un criadero tomado aisladamente posee un valor como cosa, pero solo tiene valor como hecho social a partir de su existencia relacional. A su vez, se considera que cada criadero aparece con características funcionales que permiten ejecutar ciertas acciones, las cuales acaban definiéndose en la vinculación con ese criadero dentro de un sistema (por ejemplo: los floreros permiten la presencia de flores frescas pero la acción del acompañamiento de un fallecido solo cobra sentido al vincularse con ese florero en un cementerio) (Santos, 2000). De esta forma, el espacio se modifica históricamente, se construye y reconstruye en torno a las necesidades y a los significados que cambian y, por lo tanto, resulta de la actividad de varias generaciones (Silva, 1997). 
Sobre los artículos analizados también resulta interesante notar que, en algunos casos, el espacio aparece concebido como un conjunto de casas particulares. En este sentido, el humano parece quedar confinado a un área específica. Nuevamente, la comparación con el espacio miltoniano permite destacar y revalorizar las capacidades del humano como productor y consumidor, como miembro de una clase social que interactúa y por lo tanto, que habita de manera dinámica (Costa y Teixeira, 1999). Así, la noción de espacio miltoniano permite enfatizar que las acciones no pueden considerarse aisladamente y que, en última instancia, lo que la persona realiza en su espacio privado no está escindido de su actuación o la acción de otros agentes en el espacio público. En esta línea argumental, el hogar deja de ser un elemento estático y cobra nuevo sentido (por ejemplo, al considerar qué objetos son necesarios y de dónde provienen para que esa casa exista y cumpla su función). La población de fijos (o en nuestro caso de hogares) cambia de edad a lo largo del tiempo y así, cada objeto cambia de significación, es decir, el sistema posee un dinamismo dado por las relaciones sociales. Estas relaciones actúan sobre la estructura espacial cambiándola sin necesidad de que los objetos sean distintos. De esta forma, consideramos valioso para poder entender los verdaderos efectos de una intervención de control intentar caracterizar a los objetos más allá de una concepción que entiende al espacio como un mero lugar. Es decir, sería interesante considerar las relaciones de los hogares (o fijos) entre sí, con los espacios comunes, y con las acciones humanas $y$, en particular, atender a los sentidos asignados a estos hogares en situaciones pragmáticas (por ejemplo, si la casa es un lugar para dormir, o es también un lugar de trabajo y estudio, o es un ámbito de violencia).

En este punto sugerimos una segunda conclusión parcial: las publicaciones analizadas, con algunas excepciones, parecen desatender a los sistemas de acciones, en tanto las actividades dentro de los hogares se desvinculan de lo que sucede en el espacio público. Al mismo tiempo, parecen presentar a los criaderos y hogares como si estuviesen desconectados entre sí, donde las acciones funcionales sobre ellos no son consideradas. Es decir, se piensan a los recipientes u objetos que pueden funcionar como criaderos como si fueran distinguibles de manera aislada para las personas del hogar y no fueran parte del propio habitar de la vida diaria.

\section{CONCLUSIONES Y PERSPECTIVAS}

Nuestro objetivo particular se dirigió a reflexionar sobre cómo se consideraba al espacio en términos teóricos en las publicaciones académicas analizadas, con interés en alguna intervención química de control de Aedes aegypti. A partir del recorrido trazado, esbozamos dos conclusiones parciales: la primera, que el espacio es recuperado bajo la noción de lugar. En ella destacamos una homogeneización tanto de los hogares como de los recipientes en torno a la presencia de Aedes aegypti. En cuanto a la segunda conclusión parcial, sugerimos que no se consideran los sistemas de acciones, ni tampoco, el modo en que estos sistemas se afectan mutuamente con los hogares o bien, con los recipientes.

En el contexto de estas conclusiones, sugerimos que la promesa de una mejora o solución al problema del dengue en las publicaciones analizadas es, por lo menos, problemática. Ello se debe a que, como observamos en las argumentaciones realizadas, estos estudios tienen asociados una noción de espacio como lugar. Es decir, omiten, sistemáticamente, la construcción de conocimiento sobre elementos de la organización social del espacio que, justamente, condicionarían la aparición de la enfermedad. En 
este sentido, Santos (2003) indica que la ausencia de un enfoque sistémico sobre el problema del dengue, que incluya los fenómenos de desarrollo y exclusión social, es un limitante para la ejecución de acciones integrales.

Esta afinidad que presenta Santos entre un abordaje sistémico del problema desde la investigación y, la realización de acciones integradas también es indicada por Czeresnia y Ribeiro (2000). Estos autores mencionan que la teoría y la práctica científicas construyen conceptos sobre el espacio y estructuran distintas formas de aprehensión y de acción sobre la realidad conforme a ello. Por lo tanto, la consecuencia del abordaje del espacio como lugar, en las publicaciones científicas analizadas, sería la habilitación de acciones sobre los territorios que se encuentran desvinculadas de sus complejidades y desigualdades. En este sentido, la demanda técnica del problema y los saberes científicos implicados, si bien no deberían ser excluidos, tendrían que incorporarse de forma situada (Haraway, 1995).

Una consecuencia nociva, dentro de los trabajos analizados, es que no incluyen el universo de los ambientes comunes como hospitales, escuelas y centros de salud, ni la participación de las personas en ellos. Tampoco se incluyen espacios privados empresariales (como por ejemplo fábricas, predios de almacenaje, gomerías) que colaboran con la elaboración y el mantenimiento de potenciales criaderos. Estas omisiones tienen numerosas implicancias, entre ellas, la más evidente es la falta de información acerca del movimiento humano, el cual puede afectar a las medidas de control, llevando a la ineficacia de estas. En consonancia, los programas de control dirigidos únicamente a zonas consideradas estáticas, cerradas y más productivas que otras puede llevar a la dispersión de la población del mosquito y la colonización de criaderos alternativos (Arunachalam et al., 2010). Por lo tanto, si el control se centrase exclusivamente sobre las viviendas particulares, tal vez, se estarían descuidando sitios potenciales de cría del mosquito que podrían eventualmente repoblar esas viviendas. Esto se reafirma por estudios de otros países que mencionan la importancia de los predios inhabitados o no residenciales en tanto se ha encontrado una abundancia del mosquito igual o mayor en ellos que en zonas habitacionales (Cotteaux-Lautard, Berenger, Fusca, Chardon, Simon y Pagès, 2013; Leal-Santos et al., 2014; dos Reis, Honório, Codeço, Magalhães, Lourenço-de-Oliveira y Barcellos, 2010; Baak-Baak et al., 2014).

Otra consecuencia derivada de no considerar la presencia de Aedes aegypti en espacios comunes como lo que encontramos en la mayoría de las publicaciones analizadas, es privilegiar (implícita o explícitamente) al individuo aislado como fuente del problema a ser resuelto. En este sentido, se habilitan soluciones puramente individuales, obturando demandas colectivas o luchas por el reconocimiento social (Gintrac, 2013). Es decir, el discurso estatal puede depositar la responsabilidad del problema en las personas que administran sus hogares particulares. Así, la promoción de la salud o educación para la población se convierte en una tarea de corrección cultural particular, en una actividad de convencimiento de los individuos para la adopción de una vida saludable y se omiten o desconocen las responsabilidades colectivas (Santos, 2003; Castiel y Álvarez-Dardet, 2010; Segata, 2017).

Por lo tanto, siguiendo la argumentación anterior, la consecuencia de la ausencia del espacio miltoniano en las publicaciones analizadas es el desconocimiento de las dinámicas de cada localidad y del valor simbólico que media la generación de acciones en la población. En esto se incluyen las escasas referencias en los artículos evaluados acerca de las dinámicas espaciales en su sentido social (movilidad y necesidad humana) que 
llevan a la aparición de los criaderos del mosquito. Como indica Milton Santos, una técnica como podría ser incluso la utilización sistemática de insecticidas, nunca es la misma para todos los territorios (Santos, 2000).

Con relación al problema del dengue y a nuestra aproximación en este trabajo es necesario realizar algunas aclaraciones. La recuperación de las proposiciones científicas en la formulación e implementación de programas contra el dengue no es lineal. Es decir, en tanto el campo científico no es homogéneo, podrían existir publicaciones científicas recuperando, por ejemplo, el espacio miltoniano y sin embargo, la existencia de estos artículos no garantizaría la presencia de políticas públicas acordes a ello. Por otra parte, no siempre es el conocimiento desarrollado en investigaciones nacionales el que permite la intervención en el territorio, sino que esta podría estar más ligada al conocimiento científico incorporado por los organismos internacionales. En este sentido, la inscripción del dengue como un problema de saneamiento ambiental y transfronterizo podrían habilitarlo como un instrumento de gestión u orden global (di Pasquo, Klier y Busan, 2018) que es ejecutado, en su forma más visible, por organismos mundiales de salud (como por ejemplo: OMS).

Otra aclaración relevante, respecto de nuestra aproximación, se dirige a señalar que el desarrollo de publicaciones científicas como las seleccionadas podría legitimar intervenciones químicas para controlar el dengue. Es decir, en tanto los estudios analizados concluyen, mayormente, con resultados positivos respecto de la utilización de ciertos controles químicos, estas investigaciones podrían utilizarse como fuentes de conocimiento para legitimar ciertos programas de prevención en los territorios. Esta transferencia directa de conocimiento en el control químico de Aedes aegypti a la aplicación en territorio es explicitada también en otros artículos de los mismos autores de los trabajos analizados (Licastro, Masuh, Seccacini, Harburguer, Lucia y Zerba, 2010). Por otro lado, Fundación Mundo Sano ha financiado y utilizado algunas de las publicaciones analizadas (Gurtler, Garelli y Coto, 2009; Garelli et al., 2009) para realizar controles de dengue en el norte de Argentina que involucran el uso de productos químicos. Este tipo de controles no está libre de cuestionamientos. Las sustancias adulticidas o larvicidas comprometen intereses mercantiles privados que se benefician de su fabricación y han provocado numerosas preguntas acerca de sus consecuencias en el ambiente y para la salud (Santos, 2003; Santos y Augusto, 2011; Parens, Bar-Yam, Morales, Nijhout y Xavier Costa, 2017; da Silva Augusto et al., 2016; OMS, 2015). Además, han generado situaciones de resistencia al químico en la población del vector y una pasividad en las acciones colectivas de prevención por parte de las comunidades y de las instituciones de gobierno.

Por otro lado, entendemos que en la producción de conocimiento científico se pueden encontrar dificultades operativas para abarcar la categoría de espacio miltoniano. Una forma de superar algunas simplificaciones metodológicas y lograr mayor integralidad en los abordajes sería superar la separación cuantitativo/cualitativo propia del pensamiento moderno. En este sentido, la triangulación de resultados es una propuesta que se ha destacado para los problemas complejos tales como aquellos derivados del cambio climático (Plowright, Sokolow, Gorman, Daszak y Foley, 2008). El proceso de triangulación implica el agrupamiento de evidencia científica acerca del sistema a través de la combinación iterativa de los resultados de investigaciones de laboratorio, de campo, de modelaje y de estudios sociales. Solo a través de la interacción de estas metodologías, propias de cada estudio, podríamos intentar comprender el proceso generativo de un 
sistema complejo y así derivar posibles intervenciones (Santos, 2003; Plowright et al., 2008). Adicionalmente, Almeida Filho (2007) propone caminos posibles para producir diseños mixtos de estudio o hibridación de datos de diferente naturaleza, que serían muy pertinentes al tipo de investigación necesaria en dengue.

La comparación realizada con el espacio miltoniano permite en este punto, sugerir ciertos aspectos prospectivos. Al respecto, la recuperación de la categoría de espacio miltoniano permitiría volver a pensar en las condiciones de cada territorio (Spinelli, 2016) como condicionantes evidentes del dengue en cada región. Esto resulta de particular relevancia en tanto las políticas públicas en torno al dengue suelen centrar sus discursos en la responsabilidad del otro (Gottero, 2013; 2015; 2016): ya sea del país limítrofe que no posee un control eficiente del vector $y$, por lo tanto, genera casos importados, en la falta de voluntad de las comunidades para cumplir con las recomendaciones sanitarias, o en los pobres que mantienen focos de enfermedad continuos.

Para finalizar, sugerimos la posibilidad de introducir modificaciones epistémicas en las diferentes ciencias involucradas, de manera tal que se avance en el conocimiento del territorio con sus particularidades y con los actores sociales que posee, que también tienen poder en las reglas del juego social (Matus, 2014). El fenómeno biológico del dengue es también social y debe ser entendido en su dependencia territorial. Con esto no queremos indicar que no existan estudios epidemiológicos que lo consideren, sino insistir en la necesidad de ello en todos los casos. El derecho al territorio (como suma de los derechos sociales) y a su pertenencia es la ciudadanía. Por lo tanto la falta de consideración por la ciudadanía a través de intervenciones despreocupadas por el territorio, podrían llevar a la permanencia de la enfermedad (Faria y Bortolozzi, 2009). Es decir, en tanto los científicos continúen simplificando la presencia de la enfermedad, los ciudadanos seguirán preguntándose por la salud en sus territorios y el problema del dengue seguirá irresuelto.

\section{AGRADECIMIENTOS}

El presente trabajo fue realizado con el subsidio PICT-2015-1815 de la Agencia Nacional de Promoción Científica y Técnica (Argentina) y con una beca doctoral financiada por el Consejo Nacional de Investigaciones Científicas y Técnicas (CONICET).

\section{REFERENCIAS}

Almeida Filho, N. (2007). Por una epidemiología con (más que) números: cómo superar la falsa oposición cuantitativo- cualitativo. Salud Colectiva, 3(3), 229.

Administración Nacional de Medicamentos, Alimentos y Tecnología ANMAT (2017). Eficacia y seguridad de la vacuna tetravalente para dengue (DengvaxiaNR). Recuperado de http://www. anmat.gov.ar/ets/ETS_DENGUE_agosto-EN2.pdf (consulta 29 de octubre de 2019).

Arunachalam, N.; Tana, S.; Espino, F.; Kittayapong, P.; Abeyewickreme, W.; Wai, K.T.; Tyagi, B.K.; Kroeger, A.; Sommerfeld, J. y Petzold, M. (2010). Eco-bio-social determinants of dengue vector breeding: a multicountry study in urban and periurban Asia. Bulletin of the world health organization, 88(3),173-184.

Baak-Baak, C.M.; Arana-Guardia, R.; Cigarroa-Toledo, N.; Loroño-Pino, M.A.; Reyes-Solis, G.; Machain-Williams, C.; Beaty, B.J.; Eisen, L. y García-Rejón, J.E. (2014). Vacant lots: productive sites for Aedes (Stegomyia) aegypti (Diptera: Culicidae) in Mérida 
City, México. Journal of Medical Entomology, 51(2), 475-483.

Breilh, J. (2010). La epidemiología crítica: una nueva forma de mirar la salud en el espacio urbano. Salud colectiva, 6(1), 83-101.

Breilh, J. (2015). Epidemiología crítica: Ciencia emancipadora e interculturalidad (1º edición). Buenos Aires: Lugar Editorial.

Castiel, L.D. y Álvarez-Dardet, C. (2010). La salud persecutoria: los límites de la responsabilidad ( $1^{\circ}$ edición en español). Buenos Aires: Lugar Editorial.

Costa, M. da C.N. y Teixeira, M. da G.L.C. (1999). A concepção de 'espaço' na investigação epidemiológica. Cadernos de Saúde Pública, 15(2), 271-279.

Cotteaux-Lautard, C.; Berenger, J.-M.; Fusca, F.; Chardon, H.; Simon, F. y Pagès, F. (2013). A new challenge for hospitals in southeast France: monitoring local populations of $\mathrm{Ae}-$ des albopictus to prevent nosocomial transmission of dengue or chikungunya. Journal of the American Mosquito Control Association, 29(1), 81-83.

Czeresnia, D. y Ribeiro, A.M. (2000). O conceito de espaço em epidemiologia: uma interpretação histórica e epistemológica. Cadernos de Saúde Pública, 16(3), 595-605.

Dantur Juri, M.J.; Zaidenberg, M. y Santana, M. (2013). The efficacy of a combined larvicide-adulticide in ultralow volume and fumigant canister formulations in controlling the dengue vector Aedes aegypti (Diptera: Culicidae) in Northwest of Argentina. Parasitology Research, 112(3), 1237-1246.

Faria, R.M. y Bortolozzi, A. (2009). Espaco, território e saúde: contribuicoes de Milton Santos para o tema da geografia da saúde no Brasil. Raega-O Espaço Geográfico em Análise, 17, 31-41.

Francese, C. y Folguera, G. (2018). Saberes simplificados, tecnociencia y omisión de riesgos. El caso de los organismos genéticamente modificados. RUNA, archivo para las ciencias del hombre, 39(2),5-27.

García, R. (2000). El conocimiento en construcción: de las formulaciones de Jean Piaget a la teoría de sistemas complejos. Barcelona: Gedisa Editorial.

Garelli, F.M.; Espinosa, M.O.; Weinberg, D.; Coto, H.D.; Gaspe, M.S. y Gurtler, R.E. (2009). Patterns of Aedes aegypti (Diptera: Culicidae) infestation and container productivity measured using pupal and Stegomyia indices in northern Argentina. Journal of Medical Entomology, 46(5), 1176-1186.

Garelli, F.M.; Espinosa, M.O.; Weinberg, D.; Trinelli, M.A. y Gürtler, R.E. (2011). Water use practices limit the effectiveness of a temephos-based Aedes aegypti larval control program in northern Argentina. PLoS Neglected Tropical Diseases, 5(3), e991.

Gintrac, C. (2013). Las aportaciones de la geografía radical y la geografía crítica anglosajona a la teoría urbana. Urban, (6), 53-61.

Gordis, L. (2015). Epidemiología. Barcelona: Elsevier España. Recuperado de https:// ebookcentral.proquest.com/lib/udl/reader.action?doclD=3429502 (consulta 29 de octubre de 2019).

Gottero, L. (2013). "Caso con antecedentes de viaje": una problematización de la causalidad construida sobre el dengue en Argentina (2009-2013). eä Journal, 4(2). Recuperado de http://www.ea-journal.com/en/numeros-anteriores/70-2014-04-06-15-57-18/612-caso-con-antecedentes-de-viaje-causalidad-dengue-2 (consulta 29 de octubre de 2019).

Gottero, L. (2015). Dengue en la agenda pública argentina: relatos institucionales sobre los brotes en la frontera noroeste (2009-2013). Punto Cero, año 20, 31(2), 21-34. 
Gottero, L. (2016). Indicadores de derechos humanos en políticas públicas contra el dengue en Argentina: un desafío pendiente. Población y Salud en Mesoamérica, 13(2). Recuperado de http://revistas.ucr.ac.cr/index.php/psm/article/view/22163 (consulta 29 de octubre de 2019).

Gürtler, R.E., Garelli, F.M. y Coto, H.D. (2009). Effects of a five-year citywide intervention program to control Aedes aegypti and prevent dengue outbreaks in northern Argentina. PLoS Neglected Tropical Diseases, 3(4), e427.

Haraway, D.J. (1995). Ciencia, cyborgs y mujeres: la reinvención de la naturaleza. Madrid: Cátedra. Harburguer, L.; Beltrán, G.; Goldberg, L.; Goldberg, L.; Zerba, E.; Licastro, S. y Masuh, H. (2011). A new strategy for Aedes aegypti (Diptera: Culicidae) control with community participation using a new fumigant formulation. Journal of Medical Entomology, 48(3), 577-583.

Leal-Santos, F.A.; Santana, M.B. de A.; Figueiredo, D.A. de; Oliveira, M.M. de; Acel, A.M.; Ribeiro, A.L.M.; Rodrigues, J.S.V.; Carvalho-Leandro, D.; Miyazaki, R.D. y Leite-Jr, D.P. (2014). Effective surveillance of vector dynamics of Aedes aegypti in a hospital setting in Cuiabá, Mato Grosso, Brazil. The Journal of Infection in Developing Countries, 8(10), 1356-1360.

Licastro, S.; Masuh, H.; Seccacini, E.; Harburguer, L.; Lucia, A. y Zerba, E. (2010). Innovación en herramientas de control del mosquito Aedes aegypti (Diptera: Culicidae), transmisor del dengue en Argentina. Ciencia e investigación, 60(4), 49-57

Lucia, A.; Harburguer, L.; Licastro, S.; Zerba, E. y Masuh, H. (2009). Efficacy of a new combined larvicidal-adulticidal ultralow volume formulation against Aedes aegypti (Diptera: Culicidae), vector of dengue. Parasitology Research, 104(5) ,1101-1107.

Luz, M.T. (1997). Natural, racional, social: razón médica y racionalidad científica moderna. Buenos Aires: Lugar Editorial.

Mastrangelo, A. (2013). "El agua no se le niega a nadie" Estudio social sobre la prevención del dengue en un barrio de Clorinda, Formosa, nordeste argentino. De Prácticas y Discursos. Cuadernos de Ciencias Sociales, 2(2), 1-19.

Masuh, H.; De Licastro, S.A.; Lopez, P.A.; Vega, C. y Zerba, E. (2003). Field evaluation of a smoke-generating formulation containing beta-cypermethrin against the dengue vector in Argentina. Journal of the American Mosquito Control Association, 19(1), 53-57.

Matus, C. (2014). Adiós, señor presidente. Buenos Aires: Editorial de la UNLa.

Meade, M.S.; Earickson, R.J. y Gesler, W. (1988). Medical Geography. New York: The Guilford Press.

Ministerio de Salud de la Nación, MSAL (2016a). Boletín Integrado de Vigilancia 327 SE 37. Recuperado de http://www.msal.gob.ar/images/stories/boletines/Boletin-Integrado-De-Vigilancia-N327-SE37.pdf (consulta 29 de octubre de 2019).

Ministerio de Salud de la Nación MSAL (2016b). Directrices para la prevención y control de Aedes aegypti. Recuperado de http://www.msal.gob.ar/images/stories/bes/graficos/0000000235cnt-01-directrices-dengue-2016.pdf (consulta 29 de octubre de 2019).

Monken, M.; Peiter, P.; Barcellos, C.; Iñiguez Rojas, L.; Navarro, M.; Gondim, G.M.M. y Gracie, R. (2008). O território na saúde: construindo referências para análises em saúde e ambiente (pp.62-68). En A.C. Miranda, C. Barcellos, J.C. Moreiro y M. Monken (Comps.) Território, ambiente e saúde. Editora Fiocruz: Rio de Janeiro.

Organización Mundial de la Salud OMS (2015). IARC Monographs Volume 112: evaluation offive 
organophosphate insecticides and herbicides. Recuperado de https://www.iarc.fr/wp-content/ uploads/2018/07/MonographVolume112-1.pdf (consulta 29 de octubre de 2019).

Organización Panamericana de la Salud, OPS (2019). Actualización Epidemiológica Dengue. Recuperado de https://www.paho.org/hq/index.php?option=com_docman\&view=download\&category_slug=dengue-2158\&alias=47785-22-de-febrero-de-2019-dengue-actualizacion-epidemiologica-1\&ltemid=270\&lang=en (consulta 29 de octubre de 2019).

Parens, R.; Bar-Yam, Y.; Morales, A.; Nijhout, H.F. y Xavier Costa, F. (2017). A possible link between pyriproxyfen and microcephaly. PLoS Currents. Recuperado de http://currents.plos. org/outbreaks/article/a-possible-link-between-pyriproxyfen-and-microcephaly/ (consulta 29 de octubre de 2019).

di Pasquo, F., Klier, G. y Busan, T. (2018). Orden mundial, hegemonía y problemática ambiental. Sociedad y Ambiente, (18), 95-116.

di Pasquo, F.; Klier, G.; Busan, T.E. y del Castillo, D. (2019). Objetividad, Ecología y Problemática Ambiental. Cultura-hombre-sociedad, 29(1), 225-248.

Plowright, R.K.; Sokolow, S.H.; Gorman, M.E.; Daszak, P. y Foley, J.E. (2008). Causal inference in disease ecology: investigating ecological drivers of disease emergence. Frontiers in Ecology and the Environment, 6(8), 420-429.

Raghavendra, K.; Barik, T.K.; Reddy, B.P.N.; Sharma, P. y Dash, A.P. (2011). Malaria vector control: from past to future. Parasitology Research, 108(4), 757-779.

Ramírez Velázquez, B.R. y López Levi, L. (2015). Espacio, paisaje, región, territorio y lugar: la diversidad en el pensamiento contemporáneo. México: UNAM, Instituto de Geografía: UAM, Xochimilco.

dos Reis, I.C.; Honório, N.A.; Codeço, C.T.; Magalhães, M. de A.F.M.; Lourenço-de-Oliveira, R. y Barcellos, C. (2010). Relevance of differentiating between residential and non-residential premises for surveillance and control of Aedes aegypti in Rio de Janeiro, Brazil. Acta Tropica, 114(1), 37-43.

Rubio, A.; Cardo, M.V.; Carbajo, A.E. y Vezzani, D. (2019). Assessment of combined tools and strategies for Aedes aegypti control with low environmental impact. Parasitology Research, 118(2), 411-420.

Salinas-López, M.A.; Soto-Rojas, V.E. y Ocampo, C.B. (2018). Costos de un programa de control del vector Aedes aegypti en municipios de Colombia: el caso de Girón y Guadalajara de Buga, 2016. Cadernos de Saúde Pública, 34(12). Recuperado de http:// www.scielo.br/scielo.php?script=sci_arttext\&pid=S0102-311X2018001205006\&ln$\mathrm{g}=\mathrm{es} \&$ tlng=es (consulta 29 de octubre de 2019).

Samaja, J. (2003). Desafíos a la epidemiología (pasos para una epidemiología 'Miltoniana'). Revista Brasileira de Epidemiologia, 6(2), 105-120.

Santos, M. (2000). La naturaleza del espacio: técnica y tiempo, razón y emoción. Barcelona: Ariel. Santos, S.L. dos (2003). Avaliação das ações de controle da dengue: aspectos críticos e percepção da população. Estudo de caso em um município do Nordeste. Tesis de Maestría. Centro de Pesquisas Aggeu Magalhães da Fundação Oswaldo Cruz/FIOCRUZ.

Santos, S.L. dos y Augusto, L.G. da S. (2011). Modelo multidimensional para o controle da dengue: uma proposta com base na reprodução social e situações de riscos. Physis: Revista de Saúde Coletiva, 21(1), 177-196.

Sartori, G. (1994). La comparación en las ciencias sociales. Madrid: Alianza Editorial. Sartori, G. (2011). La política: lógica y método en las ciencias sociales. México: Fondo de Cul- 
tura Económica.

Seccacini, E.; Lucia, A.; Harburguer, L.; Zerba, E.; Licastro, S. y Masuh, H. (2008). Effectiveness of pyriproxyfen and diflubenzuron formulations as larvicides against Aedes aegypti. Journal of American Mosquito Control Association, 24(3), 398-403.

Seccacini, E.; Juan, L.; Zerba, E. y Licastro, S. (2014). Aedes aegypti (Diptera: Culicidae): evaluation of natural long-lasting materials containing pyriproxyfen to improve control strategies. Parasitology Research, 113(9), 3355-3360.

Segata, J. (2017). O Aedes aegypti e o digital. Horizontes Antropológicos, 23(48), 19-48.

da Silva Augusto, L.G.; Gurgel, A.M.; Costa, A.M.; Diderichsen, F.; Lacaz, F.A.; Parra-Henao, G.; Rigotto, R.M.; Nodari, R. y Santos, S.L. (2016). Aedes aegypti control in Brazil. The Lancet, 387(10023), 1052-1053.

Silva, L.J. da (1997). O conceito de espaço na epidemiologia das doenças infecciosas. Cadernos de Saúde Pública, 13(4), 585-593.

Spinelli, H. (2016). Volver a pensar en salud: programas y territorios. Salud Colectiva, 12(2), 149-171.

World Health Organization WHO (Organización Mundial de la Salud) (2017). Updated Questions and Answers related to information presented in the Sanofi Pasteur press release on 30 November 2017 with regards to the dengue vaccine Dengvaxia. Recuperado de https://www. who.int/immunization/diseases/dengue/q_and_a_dengue_vaccine_dengvaxia/en/ (consulta 29 de octubre de 2019).

Zusman, P. (2002). Milton Santos. Su legado teórico y existencial (1926-2001). Documents d'Anàlisi Geogràfica, 40, 205-222.

Carolina Ocampo es Licenciada en Ciencias Biológicas. Becaria Doctoral del Consejo Nacional de Investigaciones Científicas y Técnicas (CONICET). De 2011 a 2014, becaria de Investigación en el marco del Convenio de Asistencia Técnica "Monitoreo de Aedes aegypti en la Ciudad de Buenos Aires" entre el Gobierno de la Ciudad y la Facultad de Ciencias Exactas y Naturales de la UBA. Becaria de investigación en el marco de la Beca CIN (Estimulo a las Vocaciones Científicas) entre 2014-2015 en el Grupo de Estudio de Mosquitos de la FCEN. Actualmente, investigación bajo el marco de la beca Conicet con los directores Aníbal Carbajo y Guillermo Folguera desde abril de 2018. La investigación se basa en una comparación en torno a la diversidad de la epidemiología para hallar argumentos epistémicos que permitan explicar el fracaso de las intervenciones en dengue en Argentina. Facultad de Ciencias Exactas y Naturales. Universidad de Buenos Aires. Intendente Güiraldes 2160 Ciudad Universitaria, (1428) Ciudad Autónoma de Buenos Aires, Buenos Aires, Argentina, caro.ocampo.mallou@gmail.com, ORCID https://orcid.org/0000-0002-1172-6910

Federico di Pasquo es Doctor en Ciencias Biológicas. Investigador Asistente del Consejo Nacional de Investigaciones Científicas y Técnicas (CONICET). Desde hace una década dedicado formalmente a la investigación en el campo de la Filosofía e Historia de las Ciencias. En particular, las investigaciones se han dirigido a la teoría de conocimiento asumida por la Ecología y a la inserción de esta disciplina en las problemáticas ambientales, destacándose la relación entre el conocimiento experto y otras formas de conocimiento. Las investigaciones se encuentran publicadas tanto en el ámbito internacional como nacional. Grupo de Filosofía de la Biología. Facultad de Ciencias Exactas y Naturales. Universidad de Buenos Aires. Intendente Güiraldes 2160 Ciudad Universitaria, (1428) Ciudad Autónoma de Buenos Aires, Buenos Aires, Argentina, dipasquof@yahoo.com.ar, ORCID https://orcid.org/0000-0001-5907-2437 
Aníbal Carbajo es Doctor en Ciencias Biológicas. Investigador Independiente del Consejo Nacional de Investigaciones Científicas y Técnicas (CONICET). Ha escrito mas de treinta publicaciones como primer autor o coautor desde 1997 hasta 2018 siempre en la línea de enfermedades transmitidas por vectores, y en particular acerca de la ecología de culícidos y roedores. Tiene cuarenta y nueve participaciones a congreso, doce informes técnicos relacionados a infestación de mosquitos y riesgo de transmisión de dengue, ocho asesorías profesionales relacionadas a Sistemas de Información Geográfica y riesgo de transmisión de dengue. Actualmente es tutor de tres investigadores de CONICET, un becario postdoctoral y dos tesistas doctorales. Dirige el Grupo de Ecología de Enfermedades Transmitidas por Vectores. Instituto de Investigación e Ingeniería Ambiental. Universidad Nacional de San Martín. Campus Miguelete, 25 de Mayo y Francia, (1650) San Martín, Buenos Aires, Argentina, acarbajo@unsam.edu.ar, ORCID https:// orcid.org/0000-0002-8792-7276 